\title{
Developing Emergent Play in Collaborative Online Experiences
}

\author{
Damian Hills \\ UTS Games Studio, University of Technology, Sydney, Australia \\ damski@assimilate.net
}

\begin{abstract}
This paper will discuss common features of emergent play in the context of developing an online collaborative practice-based research project, assimilate. Emergent play features, such as development of fictional worlds will be identified, followed by a discussion of player experience of emergent play. The paper proposes an system framework that invites narrative emergent play facilitated by a set of clearly defined and simplified affordances that provide recognisable metaphors for collaboration.
\end{abstract}

Keywords: Interaction Design, Creativity Support Tool, Interactive Digital Storytelling, Narrative Intelligence, Conversational Information System, Cybernetics.

\section{Introduction}

Recent developments [1] in web application technology have developed appropriate research areas for experimentation in emergent play. The technology has allowed for server architectures to asynchronously support rich interface to develop new and interesting entertainment platforms. This is of special interest to developers and researchers interested in novel experiments for emergent play and collaboration.

This paper will discuss common features of emergent play in the context of developing an online collaborative practice-based research project, assimilate [2]. (Figure 1).

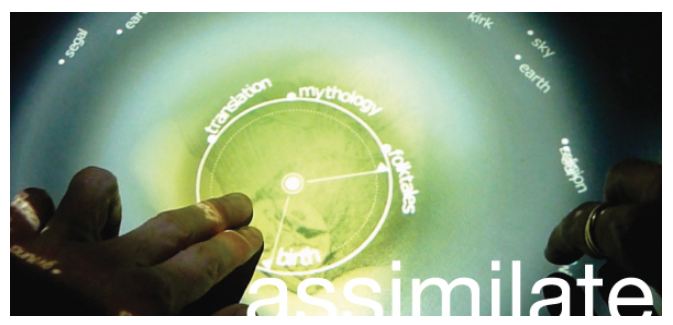

Fig. 1. Assimilate interface

The project is a software design for collaborative narrative construction adapted from previous designs for online collaboration. Emergent play features, such as 
development of fictional worlds will be discussed, followed by a discussion of player experience of emergent play. Finally, an outline of the project with appropriate analysis of how these features are applicable.

\section{Interface}

Emergence in games is considered a fundamental game feature where collections of simple rules gives rise to new variations on behavior or outcomes. [3] In the context of play within games and other interactive media including playable games [4], emergence is considered the most appealing of designs. As it allows for such narrative possibilities as re-playability and player generated creativity with full agency and immersive qualities.

Given the established problems with narrative and player agency [5] researchers are identifying common design features that generate emergent play. These can be considered applicable to both games and play, as the notable differences in how a narrative coherence is generated, either directed by top down processes, such as a drama management [6] or a bottom-up driven experience with the latter as the design goal of most desirability.

Two notable features will be discussed here, firstly the identification of boundaries that develop narrative coherence including fictional worlds, story worlds or contextual frames that place limits on emergence. Following this, the player experience of narrative emergence and possible solutions to maintaining collaborative engagement through an improvisational play.

\subsection{Fictional World Coherence}

Researchers of emergent narrative and play for digital experiences identify the development of the fictional world as basis for developing player or character based generated narratives. The experience of narrative differs greatly to the perception of narrative within films. For example, temporal events in games connects the play time, or discourse of the narrative (the sequence of events) directly with fictional time, or how the events are told [7]. Game narrative becomes problematic when presenting fictional time in any achronological order commonly seen in films or fiction. Narrative is rather a direct consequence of player action and not perceived as the action evolves. This paradox of narrative comprehension in games has shifted research into understanding how narrative emergence may develop directly in fictional worlds.

Games excel at the development of fictional worlds providing player agency and emergent play [8] with rich story-building features constructed by player activities. They contain spatial metaphors that develop narrative possibilities suggesting interfaces may play a prominent role in player developed narratives. Fictional world metaphors are linked with aspects of the real world that exhibit recognisable, familiar activities and also places boundaries on context. Defining contextual boundaries [9] contains the possibility space for emergence and maintains coherence through 
limitation. For a consistent and coherent world this is necessity as the link between emergent play, story and social world is deeply intertwined [10]. A play boundary in this case may just be an implicit or innate agreement on how to act, such as a 'magic circle' [11] or player attitude [12] or by placing specific design limitations on behavior to contain emergence [13].

Fictional worlds that aim for coherence should be designed to develop implicit events [14] rather than explicitly authoring sequences that will potentially exhaust the system of interesting outcomes. A good example of this can be seen in Minecraft [15], a successful player driven fictional world where activities mirror aspects of real world construction. However the true success of the Minecraft experience is largely dependant on social aspects of co-creativity and community engagement. For this reason, emergent fictional worlds need the drivers of collaboration and community to develop creative potential.

\subsection{Player Experience and Participation}

The narrative paradox as outlined by [16] and others suggests emergent play and narrative would benefit from a character based model where specific behaviors may be interpreted by the player and a narrative induced from these collective environmental cues. This presents further issues in maintaining narrative comprehension as it requires the active involvement of the player in the experience.

Players of emergent experiences are heavily dependant on the act of co-creation and collaboration for emergent narrative to be recognised at a macro level. Such an experience would require the player to act in similar ways to an improvisational actor with some understanding of narrative consequences based on actions of themselves and others. This has been discussed [17] [18] [19] and further evaluated as a possible scenario [20] of emergent development of interactive digital narrative. The findings conclude that actors develop shared mental models and cognitive converge over specific dramatic performances that drive narrative forward.

Cognitive patterns such as these could be useful for understanding design for emergent play with actors who have the appropriate concern for the mutual progression of the narrative scenario. However the issue is that players are generally not trained as improvisational actors nor are they necessarily open to cues for collaboration. For design of emergent play, providing these cues can equip players with the tools they need to perform more like actors sensitive to the narrative context at hand. As such, developing interface metaphors that describe or explain the simple actions possible in the fictional environment in contrast with the familiar actions in the real world, can demonstrate how to act accordingly. This type of metaphor can also be described as an affordance [21] and provides an innate understanding of how to perform within the environment.

Players perceive affordances they see in the fictional world and understand them through the affordances they commonly associate with the real world [22]. In other words, they import the knowledge from their interaction of the real environment to understand how to act in the fictional world. This is akin to a conversation where embodiment, or innate knowledge of how the body acts within the world, can be 
transferred or hybridised with the game world. This notion of an innate affordance stems from the theory of situated and embodied cognition [23] [24], that states our bodies share in part responsibility for the way we comprehend the environment. This theory has already had some impact in the fields of human-computer interface (HCI) [25] and is further discussed for its applicability to game play and player experience [22] [26] [27]. Situated cognition also has interesting implications for emergent game design and especially relates to aspects of interaction design and online player experiences. Researchers are evaluating the possibility of developing appropriate interface mechanics to enhance emergent play and narrative [28] [29]. Designing appropriate affordances will encourage players to perform more like actors and develop a level of collaborative engagement that produces more variations of behavior and novel outcomes. Further studies into motivations and aspects of playstyle with emergent games [30] would also further techniques on how to develop such affordances.

\section{Emergent Model - Assimilate}

The following section presents the application of emergent play features for the assimilate system. The description includes an understanding of boundaries in narrative context and fictional world construction, followed by a holistic view of player experience with emergence and how interface mechanics support this collaboration for online deployment.

\subsection{Developing Coherence with Simple Rules}

The model [31] is based on Conversation Theory (CT) [32], a cybernetic theory of learning and social interaction, that self-organises a set thematic relationships based on group conversation with eventual agreement on the context and meanings. As is applicable to an emergent system, relationships are arranged from simple rules or formalisms that scale into larger networks of meaning and may be combined or pruned through player participation. These networks, known as entailment meshes [33] (Fig 2.), are based on a simple formalism such that each concept is interdependent on at least two others.

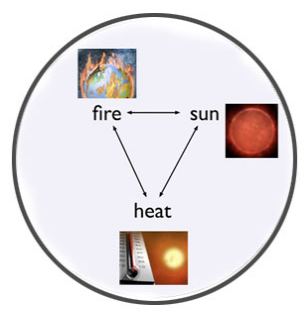

Fig. 2. Cyclic entailment relationship 
The most fundamental mesh would consists of three concepts, with every concept drawing its meaning based on its relationship to the other two. With all three exhibiting this relationship, this is said to achieve coherence within the CT theory. Coherence in this case meaning a participant can begin with any concept in the mesh and shift procedurally through the relationships in any arbitrary direction while developing an understanding of the assembled themes. This freedom to shift within an assembled concept network provides numerous entry points or cyclicity, such that there are no 'dead ends' in the entailment structure. When entailment networks are scaled or merged through collaborative action this provides the flexibility in the narrative structure and allows each participant to formulate their own point of view while developing a mental model that others may share based on their own actions within the network.

\subsection{Interface Metaphors for Emergent Conversation}

Emergent play experiences can directly benefit from collaborative experiences and the development of schemes for co-creation of artifacts within the fictional world that build community and social development.

For online play experiences the boundaries of context in the fictional space are embedded in the metaphors of the interface. This extends the conversational process and shows aspects of intention surrounding the collective narrative construction [34].

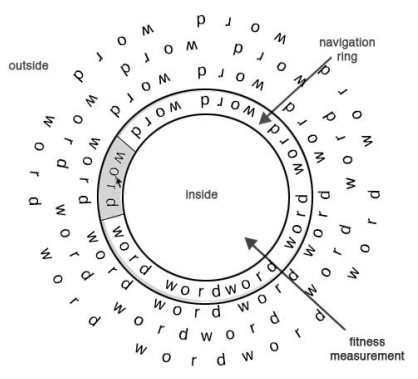

Fig. 3. Fitness measurement

Interface metaphors can support narrative comprehension and fictional world coherence through reflecting actions that are recognisable by other players and through revealing features of the system's inner processes.

Each player begins the experience by opening a 'ring' space and inputting a keyword that performs an internet search request. Retrieved video content circles the ring along with an associated annotated word tag. Salient or 'fit' tags (Fig 3.) are placed inside the ring and associated video content plays out within the ringed space in a sequence.

Tags that are outside the ring may be selected and dragged inside forming new sequences. The ring exhibits a physics model that responds to gestural movement, that may be expanded or reduced to narrow the amount of sequenced content. 
Furthermore, each player's ring may be merged with others to form new combined sequences.

These interface mechanics are considered metaphors for narrative construction and maintain common recognizable aspects of conversation associated with storytelling, as such scope, exchanging views and merging ideas. (Fig. 4) These are either performed intentionally or inadvertently through the mechanics or player actions to produce dramatic or unexpected outcomes, a common feature of narrative emergence.

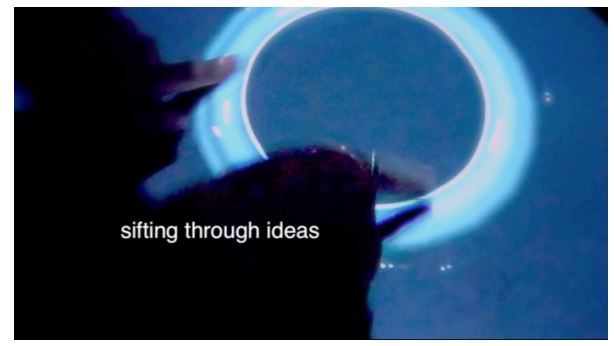

Fig. 4. Narrative Metaphors

The player rings are considered as agents in the experience that attempt to incorporate participants' creative actions and also show the self-organising conversational process. This is an attempt to visualise the system process, a form of expressive processing [35] that develops the relationship between system processes and the surface level interaction of the players. This relationship is central to idea of developing emergent play as it provides affordances to the player for enacting a conversation. In similar ways to enabling a group of improvisational actors, it exhibits clues on how actions are taking place in the fictional world and invites collaboration. This allows the player to enact a conversation with the fictional world and assume a dynamic role of narrator, player or observer.

The general approach to the interface and its incorporation with the system processes, looks to holistic paradigm for how mental models and cognitive convergence can take place. While he system formalises some narrative structure through self-organisation, the interface metaphors allow for creative possibilites. The combination of the fictional world, the interface affordances and the understanding of the player's mental model in relationship to the other players creates the possibility for an emergence to take place.

\section{Discussion}

This paper has presented a view of emergent play that considers players experience of real world knowledge to develop appropriate fictional world affordances. Our comprehension of the real world is a continual sense-making process that accumulates knowledge from the world based on our bodily relationship to the world. This takes into account a holistic view of how emergence occurs in the real world and its possible application in game worlds to drive narrative coherence. 
A study of situated and embodied cognition can provide design clues for how players may perceive certain affordances. One such view explains how our tendency to side with simple narrative explanations for complex emergent behavior. However there is an inherent disconnect between the causal relationships at the emergent level, and the eventual narrative that is extracted [36]. From this view the possibility is that layering top down approaches, such as drama management over the emergent behavioral layer (agent behaviors) only serves to replace or simplify a chaotic system of possible micro-stories rendering the system sparse of creative possibilities.

One possible solution suggested in the paper is an interface that invites play as an improvisational actor with shared goals that are developed by visible and real embodied action of the players. This is facilitated by a set of clearly defined and simplified affordances that provide recognisable metaphors for collaboration.

In terms of narrative generation, this system model is clearly hit and miss and may not be applicable for a game market that requires player expectations to be met in most, if not all circumstances. However with online web applications this is perhaps not such a great requirement as players attitudes to gaming may very well shift with respect to browser based and casual games. For this reason emergence in online web applications may provide an interesting area for further research.

\section{References}

1. Marrin, C.: Webgl specification. Khronos WebGL Working Group (2011)

2. Hills, D., Van, T., Edmonds, E., Knight, A.: Collaborative Systems for Narrative Construction (2011)

3. Juul, J.: The Open and the Closed: Games of Emergence and Games of Progression (2006)

4. Ryan, M.-L.: From Narrative Games to Playable Stories: Toward a Poetics of Interactive Narrative. StoryWorlds: A Journal of Narrative Studies 1, 43-59 (2009)

5. Louchart, S., Aylett, R.: Emergent narrative, requirements and high-level architecture. In: Proceedings of the 3rd Hellenic Conference (2004)

6. Swartjes, I., Theune, M.: A Fabula Model for Emergent Narrative. In: Göbel, S., Malkewitz, R., Iurgel, I. (eds.) TIDSE 2006. LNCS, vol. 4326, pp. 49-60. Springer, Heidelberg (2006)

7. Juul, J.: Half-Real: Video Games Between Real Rules and Fictional Worlds. MIT Press (2011)

8. Jenkins, H.: Game design as narrative architecture. Computer (2004)

9. Suttie, N., Louchart, S., Aylett, R., Lim, T.: Theoretical Considerations towards Authoring Emergent Narrative. In: Koenitz, H., Sezen, T.I., Ferri, G., Haahr, M., Sezen, D., Çatak, G. (eds.) ICIDS 2013. LNCS, vol. 8230, pp. 205-216. Springer, Heidelberg (2013)

10. Pearce, C.: A study of emergent behaviour in online games and virtual worlds (2007)

11. Salen, K., Zimmerman, E.: Rules of Play: Game Design Fundamentals. MIT Press (2004)

12. Suits, B., Hurka, T.: The grasshopper: Games, life and utopia (2005)

13. Eladhari, M.P.: AI-Based Game Design: Enabling New Playable Experiences (2011)

14. Spierling, U.: Adding Aspects of "Implicit Creation" to the Authoring Process in Interactive Storytelling. In: Cavazza, M., Donikian, S. (eds.) ICVS 2007. LNCS, vol. 4871, pp. 13-25. Springer, Heidelberg (2007)

15. Persson, M.: Minecraft (2009) 
16. Louchart, S., Aylett, R., Kriegel, M., Dias, J.: Authoring emergent narrative-based games. Journal of Game. (2008)

17. Louchart, S., Aylett, R.: Narrative theory and emergent interactive narrative. International Journal of Continuing Engineering Education and Lifelong Learning 14 (2004)

18. Swartjes, I., Theune, M.: An Experiment in Improvised Interactive Drama. In: Nijholt, A., Reidsma, D., Hondorp, H. (eds.) INTETAIN 2009. LNICST, vol. 9, pp. 234-239. Springer, Heidelberg (2009)

19. Fuller, D., Magerko, B.: Shared mental models in improvisational performance. In: Proceedings of the Intelligent Narrative Technologies III Workshop, p. 15. ACM (2010)

20. Magerko, B., Manzoul, W., Riedl, M., Baumer, A., Fuller, D., Luther, K., Pearce, C.: An empirical study of cognition and theatrical improvisation. In: Proceedings of the Seventh ACM Conference on Creativity and Cognition, pp. 117-126. ACM (2009)

21. Gibson, J.: The concept of affordances. Perceiving, acting, and knowing (1977)

22. Gee, J.P.: Video Games and Embodiment. Games and Culture 3, 253-263 (2008)

23. Hutchins, E.: Cognition in the Wild. MIT Press, Cambridge (1995)

24. Clark, A., Chalmers, D.: The Extended Mind. Analysis 58, 7-19 (1998)

25. Dourish, P.: Where the action is: The foundations of embodied interaction (2004)

26. Arjoranta, J.: Understanding Player Interpretation: An Embodied Approach (2013)

27. Rambusch, J.: The embodied and situated nature of computer game play (2006)

28. Freeman, D., La Pierre, N., Chevalier, F., Reilly, D.: Tweetris: A Study of Whole-body Interaction During a Public Art Event. In: Proceedings of the 9th ACM Conference on Creativity \&\#38; Cognition, pp. 224-233. ACM, New York (2013)

29. Alofs, T., Theune, M., Swartjes, I.: A Tabletop Board Game Interface for Multi- User Interaction with a Storytelling System. In: Camurri, A., Costa, C. (eds.) INTETAIN 2011. LNICST, vol. 78, pp. 123-128. Springer, Heidelberg (2012)

30. Canossa, A.: Give me a Reason to Dig: Qualitative Associations Between Player Behavior in Minecraft and Life Motives (2012)

31. Hills, D.: A Conversational Framework for Emergent Collaborative Storytelling. In: Proceedings of the Intelligent Narrative Technologies III Workshop, pp. 1:1-1:4. ACM, New York (2010)

32. Pask, G.: Conversation, cognition and learning: A cybernetic theory and methodology. Elsevier, Amsterdam (1975)

33. Pask, G.: Conversation theory: Applications in education and epistemology. Elsevier, Amsterdam (1976)

34. Hills, D.: Collaborative Systems for Narrative Construction. In: Edmonds, E., Candy, L. (eds.) Interacting: Art, Research and the Creative Practitioner (2011), http: //dora.dmu.ac.uk

35. Wardrip-Fruin, N.: Expressive Processing: On Process-Intensive Literature and Digital Media (2006)

36. Walsh, R.: Emergent Narrative in Interactive Media. Narrative 19, $72-85$ (2011) 